\title{
A quick analysis in relation to the electronic commerce of performance- and image- enhancing drugs based on the infrastructure of the world wide web
}

\author{
Ahmed M. Lutfi ${ }^{1,2}$ \\ ${ }^{1}$ Doctorate student (PhD), School of Life and Medical Sciences, University of Hertfordshire, Hatfield, United Kingdom, \\ ${ }^{2}$ Lecturer \& Researcher, Faculty of Medicine, University of Baghdad, Iraq
}

Aims and Objectives: Performance- and Image-Enhancing Drugs (PIEDs) refer to all known forms of substances, that can enhance either the morphology or the physiological performance or both simultaneously. The exponential rise of electronic commerce (e-commerce) for PIEDs is a major public issue, for which control protocols are to be deployed. Materials and Methods: It would be a waste of time and resources to track and/or shut down all PIED-promoting websites one by one. Cyberspace is vast; the PIED "product managers" will always adapt to surveillance-control policies over their illegitimate online businesses. A more rational approach would be to track, challenge, and tackle the same resources upon which PIED electronic commerce is based: the infrastructure of the World Wide Web (the Internet). Results: Concerning PIED e-commerce, the main resources are Google and AOL (search engines); YouTube, Wikipedia, and Facebook (social media sites); and Alibaba, Amazon, and eBay (major e-commerce websites). Conclusion: Illegal PIED e-commerce became a major public problem. The major drivers are the Internet search engines, social media sites, and major e-commerce websites. Effective protocols toward these resources would hinder any future progress of this illegitimate worldwide phenomenon.

Keywords: PIEDs, Illegitimate, Search Engines, E-Commerce, Social Media
http://nepjol.info/index.php/AJMS DOI: 10.3126/ajms.v7i4.13977 E-ISSN: 2091-0576 P-ISSN: 2467-9100

\section{INTRODUCTION}

Performance- and Imaging-Enhancing Drugs (PIEDs) refer to all existing forms of substances, natural or synthetic, that can enhance the image and/or physiologic performance of an individual. Individuals who seek PIEDs can easily conduct a simple Internet search for the purpose of information retrieval and/or product purchase, and the parameters and methods of conducting such searches are extremely vast and elaborate. ${ }^{1,2}$

\section{MATERIALS AND METHODS}

Cyberspace has become endless, and the PIED "product promoters and managers" are continuously adapting to the existing surveillance-control policies over their illegitimate form of e-commerce. PIED e-commerce has become an epidemic. Therefore, its main drivers must be traced, localized, analyzed, and eventually controlled and dismantled in an efficient manner. These resources interact in a non-hierarchical fashion. However, the search engines of the World Wide Web (Internet) rank first; the other two are social media sites and major e-commerce websites.

It is best to track these sources in a systematic way, which requires intensive and thoughtful collaboration with other departments and task forces. Professional Information Technology (IT) specialists, statistics experts, and specialist psychologistsareat the center of such interaction. 


\section{RESULTS}

It is certain that the online methods PIED seekers and consumers utilize when seeking PIEDs are highly diverse. These are influenced by geographic location, age, and gender parameters as well as religious, social, economic, cultural, and other demographic parameters.

1. Concerning the search engines, the most popular engines are Google, Yahoo!, Bing, Baidu, AOL, Ask and Lycos (Figure 1). The popularity of these engines will also vary even within the European Union itself. Globally, as of September 2015 and October 2015, Google's market share had reached $67.49 \%$ and $69.24 \%$ respectively. ${ }^{3}$

It is essential to understand how search engines work. Each particular engine displays a different list of results for an entered keyword(s), also known as a "Search Engine Results Page" (SERP). SERPs will differ in terms of total number, sequence of listed SERPs, and displayed language (original website language). It is important to know that some SERPs may not even relate to the utilized keyword/phrase, which can be attributed to the faulty indexing and data retrieval of the engine's crawler software. ${ }^{4}$

Perhaps, an effective approach would be to collaborate with the most popular search engines' officials (Google, Yahoo, and Bing) to provide log statistics concerning the PIED searches conducted by users. The statistics should provide detailed data on the utilized keywords, the number of SERPs per utilized keyword, and other critical parameters. The statistical data gained from such collaboration should be continuously updated due to the fact that each search engine utilizes "web crawler(s)" software. These crawlers function to keep the engine's indexed directories up-to-date. Unfortunately, most search engine companies will not allow disclosure of their logs. To get an idea of this aspect, in 2001, a study from the "Excite" search engine revealed the following: ${ }^{5-9}$

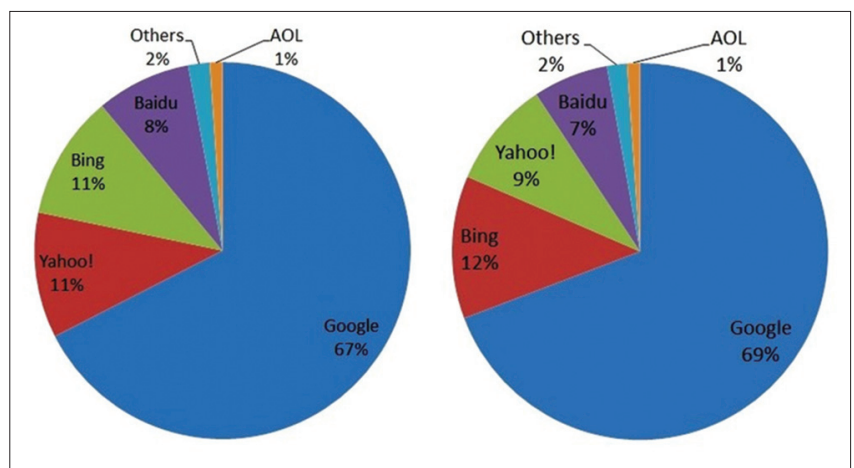

Figure 1: Search engines' market share, September 2015 (right) and October 2015 (left) $^{4}$
- The average length of search queries was 2.4 words

- The top four used terms were (empty search), and, of, and sex

- $50 \%$ of users examined the first two pages of results (with 10 results per page)

- $\quad 5 \%$ of users used advanced search options (including Boolean operators).

This study (conducted based on data collected in the period from November 102015 to November 192015 during an Internet search from Iraq using EarthLink Telecommunication (the most popular Internet service provider in Baghdad, Iraq)) was conducted across five of the engines (most popular globally). One keyword (or quoted phrase) per topic per search engine was used (Table 1, Figure 2). For each engine, the same keyword/ phrase produced variations in the total number of SERPs and in the sequence of displayed SERPs. It is worth mentioning that the "Baidu" engine, which is Chinese, can be translated to English with Google translate, but the translation is not always accurate. Therefore, data obtained from this search engine was considered confounding and hence omitted.

Moreover, when the keyword "sexual performance" was used in the "Bing" search engine, no results appeared due to an anti-pornography policy utilized by that engine (when the search was conducted from Iraq). Hence, another parameter comes into consideration, which is the geographic location from which the PIED search is conducted. Accordingly, using the same keyword/phrase in the given engine from different countries will produce a different number and sequence of displayed SERPs. Additionally, it is important to emphasize the date on which the PIED search was conducted, assearch engines' indices are continuously and rapidly updated with specialized "crawler" software(s).

When seeking to trace particular PIEDs' promotors/ distributors, it is essential to study the nature of the search engine most commonly used in that geographic location, the type of sought PIEDs, types of PIED seekers/consumers, geographic location, regional language, and the regional Internet-policing protocols. The intelligence of PIED seekers/consumers is an important factor; most individuals will follow common and convergent modalities in their pursuit of information and/or the acquisition of particular PIEDs. For example, "Wikipedia," which is always on the top of most search engines, is ranked first as a source for most Internet users. On the other hand, people of superior intelligence may seek more guaranteed methods based on data gathered from PIED users, product reviews, more accurate/secure engines, and more secure websites (with an encrypted Secure Sockets Layer (SSL), https://protocol, guaranteed 
confidentiality (e.g., Internet Protocol (IP) masking software) and seek speedy product delivery. In a similar fashion, advanced Internet search options/filters, unique to each engine, will also create SERP variations. Other factors that will induce SERP variations include keyword combinations, keyword language, use of Boolean operators including parentheses and quotations, spelling errors, nonprofessional terminology, and secret terminology for particular PIEDs.

2. Concerning the most popular e-commerce website, the "Alibaba" Chinese e-commerce website was ranked first, ahead of both Amazon and eBay combined (Table 2, Figure 3).“'On its way to becoming the world's biggest economy, China is passing another landmark. Its e-commerce market is overtaking that of America.

\begin{tabular}{|c|c|c|c|c|c|c|}
\hline & $\begin{array}{l}\text { "Weight loss } \\
\text { products" }\end{array}$ & $\begin{array}{l}\text { "Skin whitening } \\
\text { products" }\end{array}$ & $\begin{array}{c}\text { "Physical } \\
\text { performance" }\end{array}$ & $\begin{array}{c}\text { "Sexual } \\
\text { performance" }\end{array}$ & $\begin{array}{c}\text { "Mental } \\
\text { performance" }\end{array}$ & "Hallucinogenic" \\
\hline Google & $2,650,000$ & 371,000 & 781,000 & 739,000 & 476,000 & 772,000 \\
\hline Yahoo! & 337,000 & 38,200 & 260,000 & 215,000 & 98,900 & 276,000 \\
\hline Bing & 878,000 & 342,000 & 692,000 & * & 390,000 & 996,000 \\
\hline Baidu & $* *$ & & & & & \\
\hline AOL & $2,350,000$ & 611,000 & 774,000 & 734,000 & 479,000 & 775,000 \\
\hline
\end{tabular}

*Anti-pornography policy (search was conducted from Baghdad, Iraq), **Chinese language

\section{Table 2: PIEDs on top three e-commerce websites (global rankings)}

\begin{tabular}{lccccc}
\hline PIEDs & $\begin{array}{c}\text { "Weight loss } \\
\text { products" }\end{array}$ & $\begin{array}{c}\text { "Skin whitening } \\
\text { products" }\end{array}$ & $\begin{array}{c}\text { "Physical } \\
\text { performance" }\end{array}$ & $\begin{array}{c}\text { "Sexual } \\
\text { performance" }\end{array}$ & $\begin{array}{c}\text { "Mental } \\
\text { performance" }\end{array}$ \\
\hline Alibaba group & 81,637 & $1,439,863$ & 9,184 & 806 & 1,265 \\
Amazon.com & 19,515 & 11,602 & 2,326 & 1,082 & 1,040 \\
eBay.com & 171 & 1,452 & 45 & 810 & 873 \\
\hline
\end{tabular}

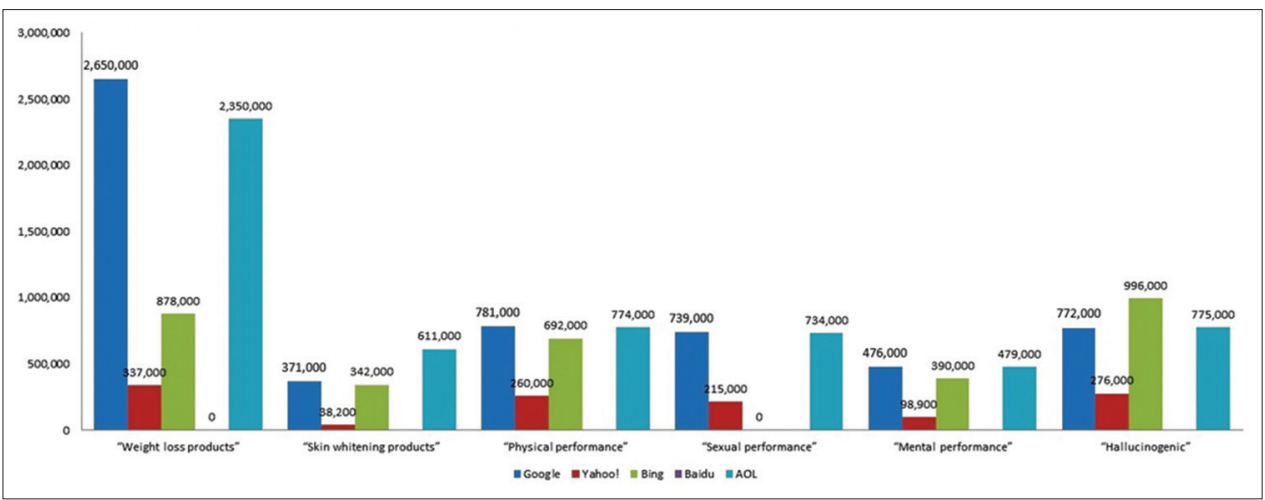

Figure 2: Bar chart, PIEDs on the most popular search engines

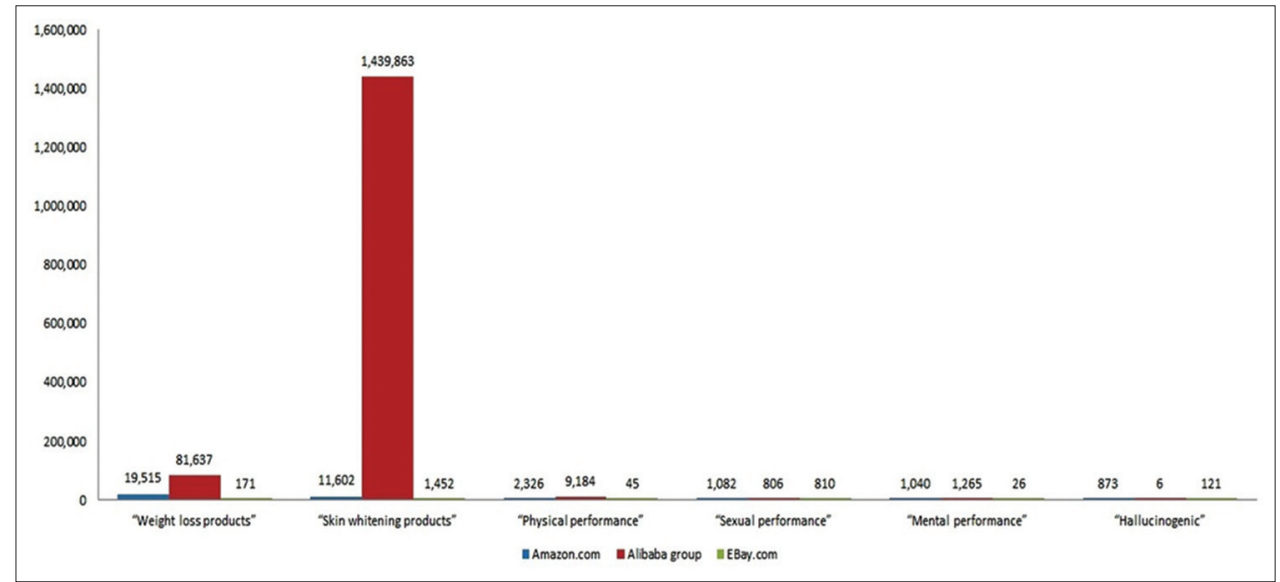

Figure 3: PIEDs on major e-commerce markets 
And one giant firm dominates the market: Alibaba, by some measures already the world's largest e-commerce company". ${ }^{10}$

3. As seen in Table 3 and Figure 4, the top social media sites are Facebook, Instagram, Twitter, LinkedIn, YouTube, and Wikipedia. ${ }^{[1]}$

\section{DISCUSSION}

To highlight statistically significant points, a correlation from Tables 1, 2, and 3 has revealed the following:

- Concerning search engines, Google and AOL produced the highest SERP numbers, while Bing and Yahoo engines ranked third and fourth, respectively. The highest SERPs were for "weight loss products" and "hallucinogenic." The lowest SERPs were for "skin whitening products."

- Concerning major e-commerce websites/markets, "Alibaba" produced the highest overall SERPs, while Amazon and eBay ranked second and third, respectively. Alibaba produced more SERPs than both Amazon and eBay combined. The top two SERP results were for "skin whitening products" and "weight loss products."The total numbers of SERPs for the other four keywords/phrases were relatively close to each other in all three e-commerce markets.

- Concerning social media, Facebook did not display a total number of SERPs but rather a continuous pageupdate of results (therefore, Facebook, in its current standards, may not be assessed using a keyword-based search technique to assess PIEDs). Surprisingly, YouTube produced the highest SERPs ("physical performance products" and "skin whitening products" ranked first and second respectively, while "weight loss products" ranked third). Wikipedia produced the highest generated SERPs for "hallucinogenic."

- As seen in Figure 4, the most popular search engines on a global scale are Google, Bing, and Yahoo. However, the top two controllers of PIED e-commerce, among search engines, are Google and AOL (both American search engines). Google was ranked first. Strangely, AOL, which was ranked fifth in search engines' global popularity (September-October 2015), ranked second in terms of total number of generated SERPs. AOL's
SERP results can be related to the nature of the crawler software implemented in the AOL search engine and the fact that AOL "offers advertisers, agencies and publishers access to AOL's online advertising tools and

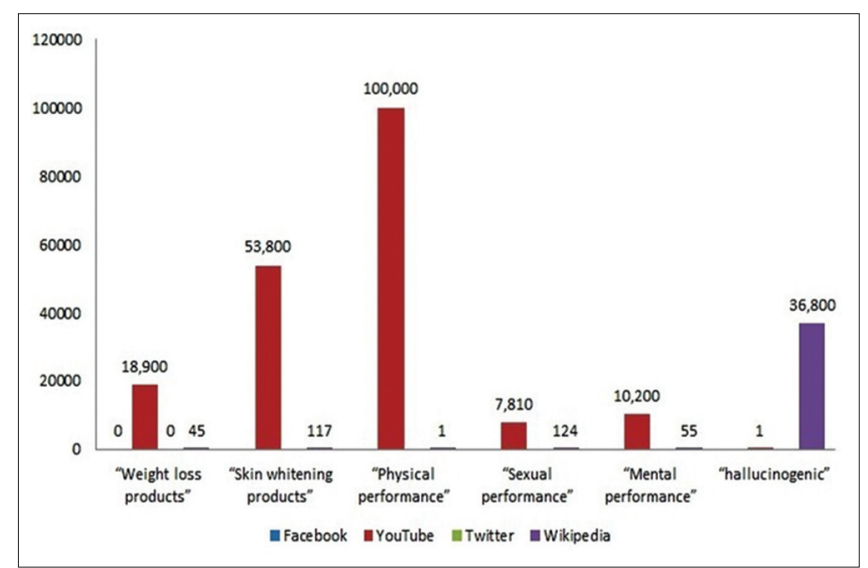

Figure 4: PIEDs on social media sites

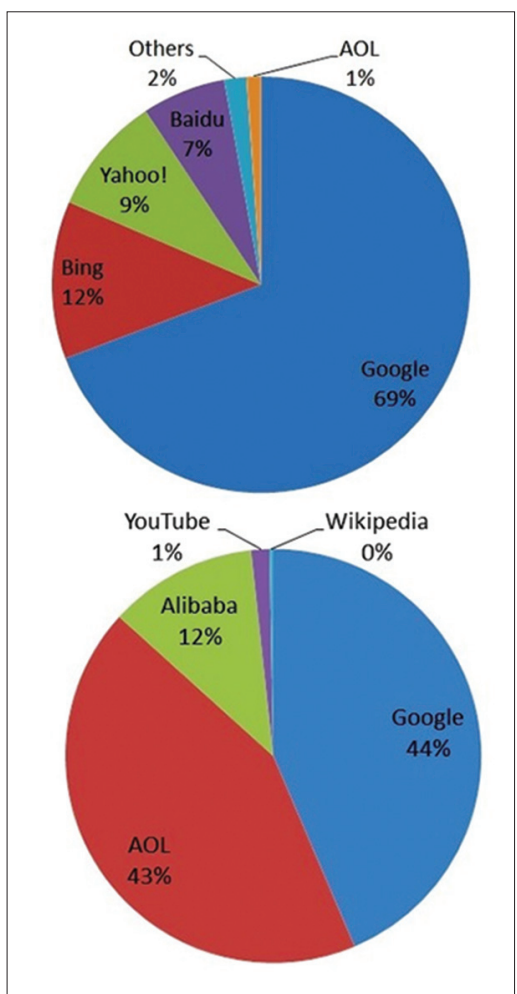

Figure 5: Most popular engines (right) vs. major controllers of PIED e-commerce (left)

\section{Table 3: PIEDs on social media sites}

\begin{tabular}{|c|c|c|c|c|c|c|}
\hline & $\begin{array}{l}\text { "Weight loss } \\
\text { products" }\end{array}$ & $\begin{array}{l}\text { "Skin whitening } \\
\text { products" }\end{array}$ & $\begin{array}{c}\text { "Physical } \\
\text { performance" }\end{array}$ & $\begin{array}{c}\text { "Sexual } \\
\text { performance" }\end{array}$ & $\begin{array}{c}\text { "Mental } \\
\text { performance" }\end{array}$ & "Hallucinogenic" \\
\hline Facebook & $\begin{array}{l}\text { n/a (does not display } \\
\text { total SERP number) }\end{array}$ & & & & & \\
\hline YouTube & 18,900 & 53,800 & 100,000 & 7,810 & 10,200 & 1 \\
\hline Twitter & n/a (inaccessible in Iraq) & & & & & \\
\hline Wikipedia & 45 & 117 & 1 & 124 & 55 & 36,800 \\
\hline
\end{tabular}


Table 4: SERPs vs. main controllers of PIED e-commerce

\begin{tabular}{ccccccc} 
& Google & AOL & YouTube & Wikipedia & Alibaba & Total \\
\hline SERPs & $5,786,000$ & $5,723,000$ & 190,711 & 37,142 & $1,532,761$ & $13,269,614$ \\
\hline
\end{tabular}

\section{Table 5: Overall correlations concluded from data in previous tables}

\begin{tabular}{|c|c|c|c|c|c|c|}
\hline & Google & AOL & YouTube & Wikipedia & Alibaba & Total \\
\hline "Weight loss products" & $2,650,000$ & $2,350,000$ & 18,900 & 45 & 81,637 & $5,100,582$ \\
\hline "Skin whitening products" & 371,000 & 611,000 & 53,800 & 117 & $1,439,863$ & $2,475,780$ \\
\hline "Physical performance" & 781,000 & 774,000 & 100,000 & 1 & 9,184 & $1,664,185$ \\
\hline "Sexual performance" & 739,000 & 734,000 & 7,810 & 124 & 806 & $1,481,740$ \\
\hline "Mental performance" & 476,000 & 479,000 & 10,200 & 55 & 1,265 & 966,520 \\
\hline "Hallucinogenic" & 772,000 & 775,000 & 1 & 36,800 & 6 & $1,583,807$ \\
\hline Total SERPs & $5,786,000$ & $5,723,000$ & 190,711 & 37,142 & $1,532,761$ & $13,269,614$ \\
\hline
\end{tabular}

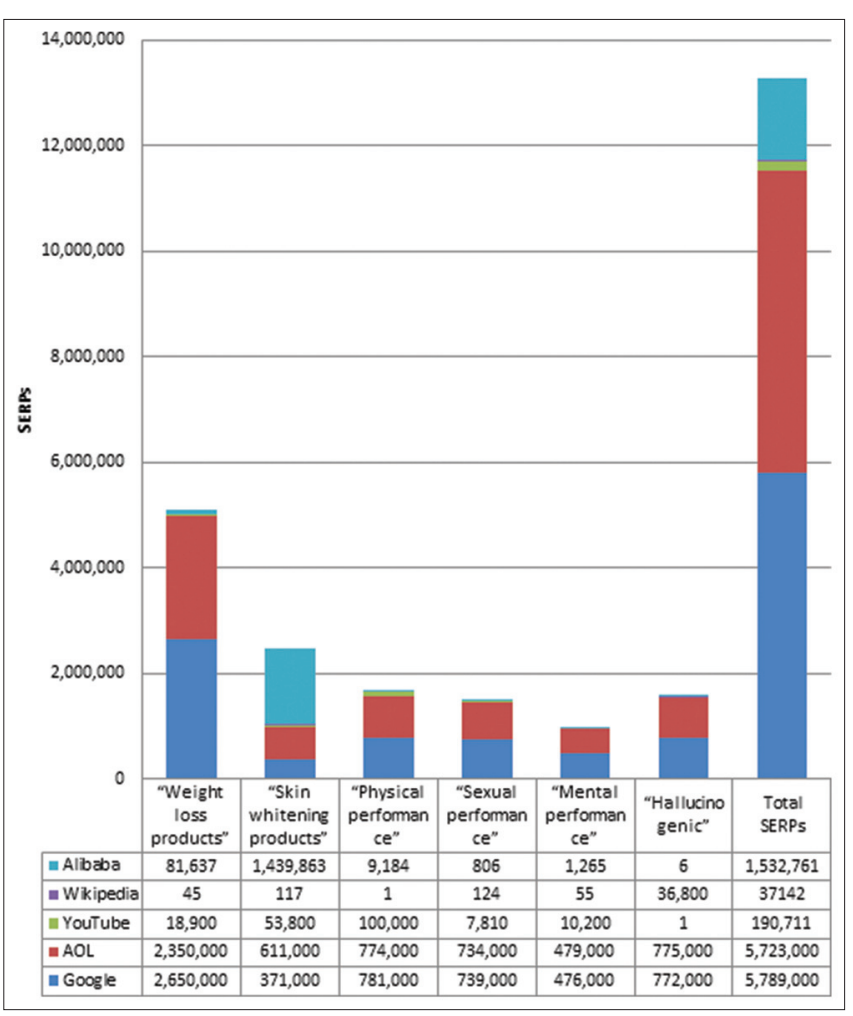

Figure 6: A component Bar chart, for analysis of PIEDs' major controllers

the ability to advertise on the original brands available through the AOL Huffington Post Media Group". ${ }^{12}$

- From the data that wereused in this quick survey, the key players (Tables 4, 5, Figures 5, 6) are Google and AOL (search engines) versus YouTube and Wikipedia (social media sites) versus Alibaba, Amazon, and eBay (e-commerce websites). The most popular keywords/phrases were "weight loss products" and "skin whitening products."

- Illegal PIED e-commerce is a critical health/financial problem. The major drivers are the Internet search engines, social media sites, and major e-commerce websites. Effective protocols toward these resources will hinder any future progress of this dangerous and illegitimate worldwide phenomenon and restrain PIED e-commerce in this era of continuously and progressively evolving communication and IT.

In the current era of fast-paced technological progression, PIED e-commerce is posing a vital and growing threat to our communities in terms of health and financial parameters. PIED promoters and distributors are continuously and intelligently evolving, using countermeasures against the e-commerce tracking/controlling policies implemented by legal organizations. Many factors control the spread of this illegal e-commerce on the Internet. The main controllers are search engines, social media sites, and major e-commerce markets/stores. Policies over these main controllers to track and control the spread of illegal PIEDs are the most rational option to utilize the exploited resources in efficiently combating PIED e-commerce.

\section{ACKNOWLEDGEMENTS}

None.

\section{REFERENCES}

1. Corazza O, Valeriani G, Bersani FS, Corkery J, Martinotti G, Bersani G, et al. "Spice," "Kryptonite," "Black Mamba": An overview of brand names and marketing strategies of novel psychoactive substances on the web. J Psychoactive Drugs 2014; 46(4):287-294.

2. Corazza O, Assi S, Simonato P, Corkery J, Bersani FS, Demetrovics, Z, et al. Promoting innovation and excellence to face the rapid diffusion of novel psychoactive substances in the EU: The outcomes of the re DNet project. Hum Psychopharmacol Clin Exp 2013; 28(4):317-323.

3. Desktop Search Engine Market Share. NetMarketShare. [online] [cited 2015 Nov 11]. Available from: https:// www.netmarketshare.com/search-engine-market-share. aspx?qprid=4\&qpcustomd $=0$.

4. Wikipedia, the free encyclopedia. Web search engine. [online] [cited 2015 Nov 14]. Available from: https://en.wikipedia.org/wiki/ Web_search_engine\#How_web_search_engines_work. 
5. Wu J, Teregowda P, Ramírez JP, Mitra P, Zheng S, and Giles CL. The evolution of a crawling strategy for an academic document search engine: whitelists and blacklists. InProceedings of the $4^{\text {th }}$ Annual ACM Web Science Conference 2012 Jun 22 (pp. 340343). ACM."

6. Kawamoto $D$ and Mills $E$. AOL apologizes for release of user search data. CNET, Aug 7, 2006.

7. Jansen BJ, Spink A, Bateman $\mathrm{J}$ and Saracevic T. Real life information retrieval: A study of user queries on the web. In ACM SIGIR Forum 1998 Apr 1 (Vol. 32, No. 1, pp. 5-17). ACM.

8. "Silverstein $\mathrm{C}$, Marais $\mathrm{H}$, Henzinger $\mathrm{M}$ and Moricz M. Analysis of a very large web search engine query log. In ACm SIGIR Forum 1999 Sep 1 (Vol. 33, No. 1, pp. 6-12). ACM.
9. Spink A, Wolfram D, Jansen MB and Saracevic T. Searching the web: The public and their queries. Journal of the American society for information science and technology 2001; 52(3):226-234

10. E-commerce in China, The Alibaba phenomenon. [online] [cited 2015 Nov 14]. Available from: http://www.economist.com/news/ leaders/21573981-chinas-e-commerce-giant-could-generateenormous-wealthprovided-countrys-rulers-leave-it.

11. Frequency of Social Media Use. [online] [cited 2015 Nov 14]. Available from: http://www.pewinternet.org/2015/01/09/ frequency-of-social-media-use-2/.

12. Wikipedia, the free encyclopedia. AOL. [cited 2015 Nov 15]. Available from: https://en.wikipedia.org/wiki/AOL\#Advertising.

Authors Contribution:

AML - 1. Study concept and design, 2. Acquisition of data, 3. Analysis and interpretation of data, 4. Drafting of the manuscript, 5. Critical revision of the manuscript for important intellectual content, 6 . Statistical analysis, 7 . Administrative, technical, and material support, 8. Study supervision

Source of Support: Nil. Conflict of Interest: None. 